\title{
Von der Neuvermessung gesellschaftlichen Wohlstands zur wohlstandsorientierten Wirtschaftspolitik
}

\begin{abstract}
Georg Feigl*
Zusammenfassung

Dieser Beitrag knüpft an die Initiativen zur Förderung einer gesellschafts- und wirtschaftspolitischen Neufokussierung auf Wohlstand und gesellschaftlichen Fortschritt an, die im Anschluss an den bahnbrechenden Bericht der Kommission zur Messung von ökonomischer Performance und gesellschaftlichem Fortschritt unter Leitung von Joseph Stiglitz entstanden ist. Ausgangspunkt ist die These, dass eine Wohlstandsorientierung in der Wirtschaftspolitik zwar adäquat ist, aber sich nicht von selbst durchsetzen wird. Es wird argumentiert, dass als Ausgangspunkt eine bessere Messung von Wohlstand und gesellschaftlichem Fortschritt sowie eine diskursive und institutionelle Verankerung wohlstandsorientierter Wirtschaftspolitik notwendig ist. Ein magisches Vieleck wohlstandsorientierter Wirtschaftspolitik wird als Bindeglied zwischen neuem Leitbild und den Wohlstandsindikatoren vorgeschlagen, um eine aktuelle Schwerpunktsetzung innerhalb von allgemein geteilten Zielen der Wirtschaftspolitik verhandelbar zu machen. Im Beitrag wird ein operationalisierter Vorschlag für Österreich umrissen. Darüber hinaus werden Ideen für eine stärkere institutionelle und diskursive Verankerung wohlstandsorientierter Wirtschaftspolitik sowie beispielhaft vier Einstiegsprojekte präsentiert, die in der aktuellen Situation im Sinne einer verstärkten Wohlstandsorientierung wirtschaftspolitisch prioritär in Angriff genommen werden sollten.
\end{abstract}

Schlagwörter: Wohlstand, Wirtschaftspolitik, Economic Governance, Fortschritt, magisches Vieleck

\section{From the Measurement of Economic Performance and Social Progress to Well-being oriented Economic Policy}

\section{Abstract}

This article follows the initiatives to foster a societal and economic re-focussing on wellbeing and social progress, which appeared after the ground-breaking report of the "Commission on the Measurement of Economic Performance and Social Progress" led by Joseph Stiglitz. Departing from the Thesis that wellbeing-orientation is adequate for economic policy making but will not be successful on its one; it is argued that a better measurement of wellbeing and social progress together with a discursively and institutionally anchoring of wellbeing-orientation within the economic governance are needed. A Magic Polygon for wellbeing-oriented economic policy is proposed to link the new paradigm with the wellbeing-indicators. Within a general framework of commonly shared objectives of economic policy, current priorities would become negotiable. This contribution sketches an operational proposal for Austria and presents ideas for a stronger institutional and discursive anchoring of wellbeing-orientation within the economic governance. Finally four entry projects are given as an example of policies which should be undertaken given the current situation within a stronger wellbeing-orientated economic governance.

Keywords: Well-being, economic policy, economic governance, prosperity, magic polygon 
Wohlstand ist der eigentliche Zweck des Wirtschaftens. Materieller - und in zunehmendem Ausmaß auch immaterieller - Wohlstand leistet einen wichtigen Beitrag zu einem guten Leben und sozialem Fortschritt. Die öffentliche Debatte und die Wirtschaftspolitik werden jedoch von einem potenziellen Mittel zum Zweck dominiert - einem möglichst hohen Bruttoinlandsprodukt (BIP) bzw. dessen jährlichen Zuwachs. In dem Maße, wie die materiellen Grundbedürfnisse gedeckt sind und BIP-Zuwächse nicht mehr breit über die gesamte Bevölkerung verteilt sind bzw. wesentliche negative gesundheitliche, soziale und ökologische Folgen nach sich ziehen, schwindet der Beitrag, den Wirtschaftswachstum für ein besseres Leben aller leistet. Weltweit gibt es deshalb Initiativen (vgl. Stiglitz/ Sen/Fitoussi 2009), die Wohlstand und Lebensqualität in den Fokus rücken. In Österreich ist es vor allem die Statistik Austria (2015), die mit dem Projekt „Wie geht's Österreich?“ eine Grundlage für die Messung von Wohlstand und gesellschaftlichem Fortschritt entwickelt.

Trotz dieser Initiativen ist die Debatte in der wirtschaftspolitischen Praxis kaum angekommen - auch nicht in Österreich (Griesser/Brand 2016). (Wirtschafts-)Wachstum ist hier nach wie vor der Referenzwert schlechthin. Eine rühmliche Ausnahme stellte zuletzt das Österreichische Wirtschaftsforschungsinstitut WIFO als einer der zentralen heimischen Akteure dar. So wird im Endbericht des großen europaweiten Projektes „WWWforEurope“ als neue strategieleitende Vision für Europa 2050 ,a region with high social and environmental standards guaranteeing its citizens a high level of wellbeing" vorgeschlagen (Aiginger 2016: 3). Ob dieses Ergebnis als singuläres Statement oder als Zeichen für ein tatsächliches Umdenken zu werten ist, wird sich u. a. daran zeigen, ob die Wirtschaftsprognosen, die die öffentliche Wahrnehmung stark prägen, weiterhin auf die Wachstumsrate als zentralen Indikator fokussieren oder ob auch hier zunehmend auf ein breiteres Set an Wohlstandsindikatoren abgestellt wird.

Als Bindeglied zwischen neuem Leitbild und Wohlstandsindikatoren braucht es allgemeine Ziele der Wirtschaftspolitik, die eine mittelfristige Ausrichtung ermöglichen. Historisch erfüllte diese Funktion das sogenannte „magische Viereck der Wirtschaftspolitik“, das in Deutschland bis heute im Grundgesetz verankert ist. Es verpflichtet die Wirtschaftspolitik auf die Ziele Wirtschaftswachstum, Vollbeschäftigung, stabile Preise und außenwirtschaftliches Gleichgewicht. In Deutschland gibt es aktuelle Bemühungen von Sebastian Dul- lien (2016) und Till van Treeck (Dullien/van Treeck 2012), die - daran angelehnt - die Wirtschaftspolitik auf eine neue Grundlage stellen wollen, ausgerichtet auf ihr übergeordnetes Ziel „Nachhaltigkeit“. Mittels 13 Indikatoren, die sie stark von internationalen Verpflichtungen oder europäischen Zielwerten ableiten, wollen sie den Fortschritt in den vier wirtschaftspolitischen „Ecken“ - materieller Wohlstand sowie ökonomische, soziale und ökologische Nachhaltigkeit - messen. Darüber hinaus schlagen Dullien und van Treeck Möglichkeiten zur strukturellen Verankerung ihres neuen magischen Vierecks nachhaltiger Wirtschaftspolitik in Deutschland vor, die von einem jährlichen Gutachten eines reformierten Sachverständigenrats bis hin zur verpflichtenden Stellungnahme der Regierung beim Regierungsantritt sowie in einem "Jahreswohlstandsbericht" anstelle des bisherigen Wirtschaftsberichts reichen.

Bei all diesen Initiativen darf jedoch nicht übersehen werden, dass die Debatte über eine Neuorientierung der Wirtschaftspolitik nur bedingt neu ist. Bereits in den 1970ern wurde sie angesichts verstärkter Verteilungskonflikte sowie der dramatischen Prognosen in Bezug auf Umweltprobleme im Bericht des Club of Rome breit geführt - in Österreich ausführlich etwa im Statistischen Zentralamt und dem damals besonders einflussreichen Beirat für Wirtschafts- und Sozialfragen (Chaloupek/Feigl 2012). Das Ergebnis blieb jedoch eher bescheiden. Die Gründe sind vielfältig, betreffen aber nur zu einem kleinen Teil methodische Schwächen (ebd.). Die wissenschaftliche Debatte sollte sich deshalb nicht nur auf konzeptionelle und methodische Fragen der Wohlstandsmessung und Bestimmung relevanter Einflussfaktoren konzentrieren, sondern Agenda-Setting bzw. die gesellschaftliche Verankerung ins Blickfeld rücken, beispielsweise mittels innovativer Formen der Einbindung neuer und alter AkteuerInnen und der Überwindung von Machtasymmetrien und nach wie vor dominanten Vorstellungen gesellschaftlichen Wohlstands (Griesser/Brand 2016: 69f.).

Ausgangspunkt für diesen Beitrag ist die These, dass eine Wohlstandsorientierung in der Wirtschaftspolitik zwar adäquat ist, aber sich nicht von selbst durchsetzen wird und sie deshalb von den wirtschaftspolitischen Akteuren getragen, institutionell verankert, adäquat gemessen und breit diskutiert werden muss, um tatsächlich handlungsleitend bzw. effektiv zu sein. Als Zielgruppe der Aktivitäten sind deshalb nicht nur ForscherInnen zu sehen, sondern auch PolitikerInnen, die öffentliche Verwaltung, Sozialpartner und andere 
zivilgesellschaftliche Gruppen sowie die breite Öffentlichkeit. Ein wichtiger Schritt wäre es demnach, neben einer besseren Messung von Wohlstand und gesellschaftlichem Fortschritt wohlstandsorientierte Wirtschaftspolitik diskursiv und institutionell zu verankern. Dieser Text möchte einen Beitrag dazu leisten.

Im ersten Abschnitt wird zunächst das Konzept des magischen Vielecks der Wirtschaftspolitik ausführlicher dargestellt. In Abschnitt 2 wird das Projekt „Wie geht's Österreich?" der Statistik Austria als konkreter Versuch einer adäquaten Messung von Wohlstand und sozialem Fortschritt skizziert. In Abschnitt 3 wird eine mögliche Verknüpfung von Zielen und Indikatoren erarbeitet, um sie gemeinsam für die Wirtschaftspolitik fruchtbar zu machen. Im darauffolgenden vierten Teil werden Ideen für eine stärkere institutionelle und diskursive Verankerung wohlstandsorientierter Wirtschaftspolitik präsentiert. In Abschnitt 5 werden beispielhaft vier Einstiegsprojekte vorgeschlagen, die in der aktuellen Situation im Sinne einer verstärkten Wohlstandsorientierung wirtschaftspolitisch prioritär in Angriff genommen werden sollten. Den Abschluss bildet eine Zusammenfassung.

\section{Das magische Vieleck als Rahmen der Wirtschafts- politik}

Wirtschaftspolitik im engeren Sinne umfasst die konkreten politischen Maßnahmen, die gesetzt werden, um wirtschaftliche Prozesse $\mathrm{zu}$ ändern oder auch anzustoßen. Diese folgen jedoch nicht nur einer rein funktionalistischen Logik des Sachzwangs, sondern Leitbildern, historischen Entwicklungspfaden, Interessen, Werten, institutionellen Vorgaben etc. Die spezifischen strukturellen Arrangements und Metadiskurse wirken auf unterschiedlichen räumlichen Ebenen strategisch-selektiv (vgl. Jessop 2014) - und beschränken somit die Bandbreite der "richtig" erscheinenden wirtschaftspolitischen Maßnahmen. Die Ausgestaltung wirtschaftspolitischer Maßnahmen ist somit nicht bloß das Ergebnis des Handelns beispielsweise der österreichischen Bundesregierung, sondern geht zunehmend darüber hinaus, insbesondere hinsichtlich der Relevanz der europäischen Ebene.

Mit dem Begriff der „Governance“ (bzw. politischen Steuerung) wird versucht, dieses strukturelle Arrangement theoretisch zu erfassen. Die Europäische Kommission definierte in ihrem Weißbuch (Europäische Kommission 2001: 5ff.), dass der Governance-Begriff für „die Regeln, Verfahren und Verhaltensweisen“ steht, „die die Art und Weise, wie ... Befugnisse ausgeübt werden, kennzeichnen, und zwar insbesondere in Bezug auf Offenheit, Partizipation, Verantwortlichkeit, Wirksamkeit und Kohärenz". In der Folge werde ich mich auf den letzten Punkt konzentrieren.

Als Paradebeispiel für die Kohärenz der wirtschaftspolitischen Steuerung auf nationaler Ebene gilt für die Vergangenheit das "magische Viereck der Wirtschaftspolitik“, das 1967 im deutschen Grundgesetz verankert wurde. Im Rahmen des Stabilitäts- und Wachstumsgesetzes wurde beschlossen, diese und zukünftige Bundesregierungen auf eine Wirtschaftspolitik zu verpflichten, die möglichst ausgewogen die übergeordneten, aber zueinander zum Teil in Konflikt stehenden Ziele Wirtschaftswachstum, Beschäftigung, Preisstabilität und außenwirtschaftliches Gleichgewicht verfolgt. Inhärente Widersprüche - beispielsweise zwischen Preisstabilität und Beschäftigung oder hohem Wirtschaftswachstum und außenwirtschaftlichem Gleichgewicht - sollten damit auch transparenter und besser verhandelbar gemacht werden. Das Gesetz steckt seither den Rahmen des deutschen Sachverständigenrates $a b$, der für die Bundesregierung die gesamtwirtschaftliche Lage darstellen und damit die Grundlage für ihre Ausrichtung schaffen soll.

In Österreich fehlt zwar eine genauere formelle Verankerung über den Grundsatz eines gesamtwirtschaftlichen Gleichgewichts hinaus, doch bildete das magische Viereck bzw. Vieleck ${ }^{1}$ auch hierzulande einen wichtigen Orientierungspunkt - gerade in Zeiten des Austrokeynesianismus (vgl. Chaloupek/Marterbauer 2008). Insbesondere in der Sozialpartnerschaft gab es den Konsens, dass Wirtschaftspolitik vor allem dann erfolgreich sein kann, wenn sie auf die Einbindung der Akteure und die Koordinierung der Ziele setzt, sodass Erwartungen stabilisiert und die Grundlage für einen Interessenausgleich geschaffen werden können.

Spätestens mit dem Beitrittsprozess zur Europäischen Union wurde die wirtschaftspolitische Governance inkohärenter. Das ist erstens ein fast zwangsläufiges Ergebnis durch die nun hinzugekommene übergeordnete europäische Entscheidungsebene, die insbesondere seit der Euro-Einführung zu einer Neuregulierung der Wirtschaftspolitik in Österreich

1 Das magische Vieleck wurde in Österreich vor allem von Kurt W. Rothschild geprägt, der das Viereck um die zusätzlichen Ziele "gerechte Einkommensverteilung“" und „Lebensqualität, gesunde Umwelt“ erweiterte (vgl. Rothschild 1996: 7). 
führte. Zweitens sind aber auch innerösterreichische Veränderungen festzustellen, wie beispielsweise die Aufwertung des Ziels eines Abbaus der Staatsverschuldung, die abnehmende Konsensbereitschaft zentraler wirtschaftspolitischer Akteure und die stärkere Orientierung auf den Export, während eine gesamtwirtschaftliche Sichtweise und hier insbesondere das Beschäftigungsziel in den Hintergrund rückte (vgl. BEIGEWUM 2015). Drittens wird die Wirtschaftspolitik auf europäischer Ebene selbst inkohärenter. Zwar findet sich in den Oberzielen im Rahmen des Vertrags über die Europäische Union in Artikel 3 noch ein reformuliertes magisches Vieleck ${ }^{2}$ mit dem übergeordneten Grundsatz das „Wohlergehen ihrer Völker zu fördern“. Die wirtschaftspolitische Ausrichtung räumt in der Praxis jedoch seit dem Vertrag von Maastricht bzw. dem vor allem zur Bekämpfung der Wirtschaftskrise 1992/93 verfassten Weißbuch „Wachstum, Wettbewerbsfähigkeit, Beschäftigung“ (Europäische Kommission 1993) „restriktiv wirkenden Zielen Vorrang“ ein (Kitzmantel 2016: 433). Spätestens mit den Refinanzierungsproblemen in Griechenland Anfang 2010 kam es zu einem verschärften Umbau des europäischen wirtschaftspolitischen Steuerungsarrangements, der auf eine stärkere Einschränkung nationaler Handlungsspielräume bzw. die Ziele Haushaltskonsolidierung und verstärkte Wettbewerbsorientierung anstelle einer ausgewogenen Wirtschaftspolitik, aber auch einen stringenteren wirtschaftspolitischen Prozess - das sogenannte Europäische Semester - abzielte (ausführlicher siehe Feigl 2014).

Eine kohärentere wirtschaftspolitische Steuerung in Österreich erfordert angesichts der Einbettung ins europäische Mehrebenensystem einerseits eine verstärkte Koordinierung in der Eurozone, andererseits aber auch nationale Schritte. Hinsichtlich des Governance-Arrangements ist die Europäische Union ein Orientierungspunkt, wenngleich der dort nur sehr beschränkte Interessenausgleich durch fehlende Einbeziehung relevanter Akteure und die weitgehend fehlende öffentliche Debatte unbedingt vermieden werden muss, wenn auf nationaler Ebene eine daran

2 Im Artikel 3 Abs. 3 heißt es: „Sie [die EU] wirkt auf die nachhaltige Entwicklung Europas auf der Grundlage eines ausgewogenen Wirtschaftswachstums und von Preisstabilität, eine in hohem Maße wettbewerbsfähige soziale Marktwirtschaft, die auf Vollbeschäftigung und sozialen Fortschritt abzielt, sowie ein hohes Maß an Umweltschutz und Verbesserung der Umweltqualität hin. Sie fördert den wissenschaftlichen und technischen Fortschritt." angelehnte Implementierung erfolgt. Für eine kohärentere ökonomische Governance nachahmenswert erscheinen folgende Punkte:

- die primärrechtlich verankerte Wohlstandsorientierung,

- ihre Konkretisierung durch grobe wirtschaftspolitische Ziele,

- der Steuerungsprozess, der eine regelmäßige Auseinandersetzung mit der gesamtwirtschaftlichen Ausrichtung sowohl im politischen System im engeren Sinn als auch der interessierten Öffentlichkeit ermöglicht,

- die Einbeziehung von ExpertInnen.

Damit sie jedoch besser funktioniert als auf europäischer Ebene und insbesondere ausgewogener gelebt wird, sind vor allem drei Änderungen relevant:

1. eine Operationalisierung der Ziele - insbesondere mit Hilfe von wohlstandsorientierten Indikatoren,

2. die Einbeziehung relevanter Akteure in die Zielformulierung,

3. eine ständige Reflexion der spezifischen Selektivitäten, die jeglicher Governance-Konzeption inhärent ist (vgl. Jessop 2014).

In einem ersten Schritt sollte ein neues magisches Vieleck der Wirtschaftspolitik formuliert werden, das Stiglitz, Sen und Fitoussi folgend auf eine breite Wohlstandsorientierung unter Berücksichtigung ökologischer und instrumenteller Ziele für eine stetige, möglichst krisenvermeidende ökonomische Entwicklung abzielt. Wichtiger Anknüpfungspunkt hierfür ist das historische magische Viereck in Deutschland inklusive der bereits in den 1970ern implizit erfolgten Erweiterungen angesichts verstärkter Verteilungskonflikte und der dramatischen Prognosen für die zukünftigen Umweltprobleme im Bericht des Club of Rome (vgl. Chaloupek/Feigl 2012; Rothschild 1996).

In Deutschland erfolgte ein erster Vorstoß in diese Richtung von Dullien und van Treeck (2012), die ein - mit dem europäischen Rahmen kompatibles - reformuliertes magisches Viereck nachhaltiger Wirtschaftspolitik inklusive eines Indikatorensets zur Überprüfung der Ziele vorschlugen. Die vier Ecken respektive Ziele bilden bei ihnen materieller Wohlstand und ökonomische Nachhaltigkeit, Nachhaltigkeit der Staatstätigkeit und der Staatsfinanzen, Soziale Nachhaltigkeit und Ökologische Nachhaltigkeit. Wirtschaftswachstum verschwindet dabei sowohl als eigenständiges Ziel als auch als Indikator (implizit ist es im daraus abgeleiteten Indikator der Produktivitätsentwicklung aber enthalten). 
Feigl: Von der Neuvermessung gesellschaftlichen Wohlstands zur wohlstandsorientierten Wirtschaftspolitik

Abbildung 1: Die dreißig das BIP ergänzenden Headline-Indikatoren von „Wie geht's Österreich?“ 2016

\begin{tabular}{|c|c|c|}
\hline Materieller Wohlstand & Lebensqualität & Umweltorientierte Nachhaltigkeit \\
\hline Arbeitsproduktivität & Armuts- oder Ausgrenzungsgefährdung & Inländischer Materialienverbrauch \\
\hline real verfügbare Haushaltseinkommen pro Kopf & Erwerbstätigenquote & Flächeninanspruchnahme \\
\hline Haushaltskonsum pro Kopf & Arbeitslosenquote & Treibhausgasemissionen \\
\hline $\begin{array}{l}\text { Einkommensentwicklung im untersten Quartil } \\
\text { der unselbstständig Beschäftigten im Vergleich } \\
\text { zum 3. Quartil }\end{array}$ & $\begin{array}{l}\text { Anteil der Personen mit (sehr) schlechter/mit- } \\
\text { tlerer/(sehr) guter subjektiver Gesundheit }\end{array}$ & Feinstaub-Emissionen \\
\hline Einkommensquintilverhältnis S80/S20 & $\begin{array}{l}\text { Anteil der 30- bis 34-Jährigen mit tertiärem } \\
\text { Bildungsabschluss }\end{array}$ & Anteil erneuerbarer Energieträger \\
\hline Nichtbezahlte Arbeit & $\begin{array}{l}\text { Anteil der Personen, die bei Problemen mit } \\
\text { Hilfe in ihrem Umfeld rechnen können }\end{array}$ & Energetischer Endverbrauch \\
\hline \multirow[t]{6}{*}{ Gender-Pay-Gap } & Freizeit in Minuten nach Geschlecht & Energieintensität \\
\hline & Subjektiv empfundene physische Unsicherheit & Energieverbrauch des Verkehrs \\
\hline & Vertrauen in das politische System & Bio- und Naturschutzflächen \\
\hline & Subjektive Umweltbelastung & Transportleistungen des LKW-Verkehrs \\
\hline & Lebenszufriedenheit & Ökosteueranteil \\
\hline & & Umsatz der Umweltwirtschaft \\
\hline
\end{tabular}

Quelle: Statistik Austria (2016), eigene Darstellung.

In Österreich wurde eine neue Version des magischen Vielecks zuletzt in der AK Wien entwickelt (siehe Feigl et al. 2014: 13). Im Vergleich zur bisher gängigen Version von Rothschild scheint Wirtschaftswachstum ebenfalls nicht mehr auf, dafür wird die Lebensqualität als eigenständiges Ziel ebenso aufgenommen wie - in Anlehnung an Dullien und van Treeck - eine stabile Staatstätigkeit.

Mehr als auf die genaue Formulierung der Ziele wird es jedoch darauf ankommen, eine breite gesellschaftliche Auseinandersetzung um die Ausrichtung der Wirtschaftspolitik anzustoßen und das magische Vieleck im wirtschaftspolitischen Steuerungsarrangement zu verankern (siehe Abschnitt 4).

\section{Wie geht's Österreich?}

Ausgangspunkt für das Projekt der Statistik Austria war der vielbeachtete Bericht einer internationalen Kommission, die sich im Auftrag des damaligen französischem Präsidenten Sarkozy mit der Beobachtung auseinandersetzen sollte, dass Zufriedenheit und Lebensqualität nicht mehr mit Wirtschaftswachstum einhergehen (vgl. Stiglitz et al. 2009: 7). Stiglitz et al. folgern daraus, dass sich der Fokus der Wirtschaftspolitik auf (materiellen) Wohlstand und Lebensqualität bzw. sozialen Fortschritt unter Berücksichtigung öko- logischer Grenzen verschieben müsse. Damit brachten sie einen Stein für unzählige Initiativen zur verbesserten Fortschrittsmessung ins Rollen, die auf internationaler wie auf nationaler Ebene begannen, an diesem Paradigmenwechsel zu arbeiten. Die bekanntesten Beispiele sind die Better-Life-Initiative der OECD (2016), die „Beyond GDP“-Aktivitäten von Eurostat (2016) und die Enquete-Kommission „Wachstum, Wohlstand, Lebensqualität“ des deutschen Bundestags (2013).

In Österreich war es vor allem die Statistik Austria, die gestützt auf die internationalen Vorarbeiten - insbesondere Stiglitz et al. und die Empfehlungen der "Sponsorship Group“ des Europäischen Statistischen Systems um Eurostat - 2012 erstmals ihren nunmehr jährlich erscheinenden unabhängigen Bericht „Wie geht's Österreich?“ (in Folge kurz WgÖ) veröffentlichte. Mittels dreißig Schlüsselindikatoren und einem Vielfachen an zum Teil nur online verfügbaren Subindikatoren, die in Orientierung an Arbeiten von Eurostat sowie der OECD unter Einbindung von Stakeholdern entwickelt wurden, soll nun die Entwicklung von Wohlstand und Fortschritt über das BIP hinaus in Österreich analysiert werden.

2017 plant die Statistik Austria vor dem Hintergrund von fünf Jahren Erfahrung eine umfassendere Neuerung bei ihren WgÖ-Aktivitäten. Wie bereits zu Beginn des Projekts wurde ein Konsultationsprozess 
mit Stakeholdern gestartet, dessen Ergebnisse allerdings zum Zeitpunkt der Fertigstellung dieses Beitrags noch nicht vorliegen.

Obwohl das Indikatorenset von Statistik Austria bereits eine weitgehend gute Ausgangsbasis für die statistische Erfassung von Wohlstand und Fortschritt in Österreich darstellt, wurde weder die laufende Dokumentation auf der Homepage noch der jährliche Bericht ein Referenzpunkt in der Öffentlichkeit, Wissenschaft oder in der wirtschaftspolitischen Debatte. Markus Griesser und Ulrich Brand (2016) untersuchten in ihrer im Herbst des Vorjahres veröffentlichten Studie zur besseren Verankerung wohlstandsorientierter Politik - wenn auch nur am Rande - die Möglichkeiten, um WgÖ mehr Gewicht zu verleihen. Einerseits seien kommunikative Maßnahmen notwendig, andererseits aber auch eine Überarbeitung des Sets mit einer besseren Aufbereitung und Legitimation, einer stärker narrativen Vermittlung der Daten sowie einer besseren Balance zwischen Komplexität und Überschaubarkeit (ebd., Tabelle 2).

Die konkrete Umsetzung dieser eher abstrakten Anforderung fehlt zwar bei Griesser und Brand, doch ist davon auszugehen, dass im Stakeholder-Konsultationsprozess einige Ideen zu finden sind. Seitens des Autors dieses Beitrags wurden folgende konkrete Vorschläge im Rahmen der Stellungnahme der AK Wien eingebracht, die zumindest zum Teil die Problembereiche adressierten:

- Die Vorgabe, lediglich bereits erhobene Daten zu verwenden, müsste aufgehoben werden, da sie sowohl thematisch (Verteilung, Arbeitswelt) als auch zeitlich (manche Schlüsselindikatoren werden bislang nicht einmal jährlich erhoben) zu wesentlichen Datenlücken führt, die einer aussagekräftigen rezenten Information widerspricht. Angesichts eines relativ geringen Ermessensspielraums von Statistik Austria würde das allerdings eine zusätzliche Finanzierung der Bundesregierung erfordern, wofür wiederum ein Bewusstsein für die Relevanz des Projektes Voraussetzung ist.

- Nicht nur eine Bewertung mittels Wettersymbolen des kurz- und langfristigen Trends in der Vergangenheit sollte bei der qualitativen Grobeinschätzung der Indikatorenentwicklung durch den ExpertInnenbeirat erfolgen, sondern auch ein Ausblick bzw. zumindest ein Überblick über die Veränderungen der Einschätzung gegenüber dem vorangegangenen Bericht. Jeder Indikator sollte dabei bewertet werden wo dies derzeit noch nicht möglich ist, sollte entweder die Grundlage geschaffen oder der Indikator durch einen anderen ersetzt werden.
- Stiglitz et al. folgend sollten Verteilungsaspekte ein stärkeres Gewicht bekommen, insbesondere im Bereich des materiellen Wohlstandes (Konsum, Vermögen), da die Entwicklung für die Bevölkerung im Durchschnitt durch stark konzentrierte Wohlstandszuwächse verzerrt werden könnte.

- Auf wenig aussagekräftige Indikatoren wie jener der Erwartbarkeit von Hilfe aus dem persönlichen Umfeld (weil absolut kaum interpretierbar und praktisch ohne Veränderung über die Zeit), der Anteil der Ökosteuern bzw. der Umsatz der Umweltwirtschaft (weil Abgrenzungsprobleme und überproportionales Wachstum umweltschädlichen Verhaltens zu einer scheinbar besseren Entwicklung führt) sollte verzichtet werden, beispielsweise durch einen Indikator für die Jobzufriedenheit, die subjektive gesellschaftliche Anerkennung oder eben zur Verteilung. So wäre es auch möglich, in allen drei Bereichen zehn Schlüsselindikatoren $\mathrm{zu}$ verwenden, die eine symbolische gleiche Gewichtung der drei Hauptbereiche signalisieren würde.

Ungeachtet dieser Verbesserungsvorschläge ist festzuhalten, dass sich in dem mehr als 200 Seiten starken Bericht bereits jetzt eine gute Datengrundlage für eine wohlstandsorientiertere Wirtschaftspolitik findet, die eine verstärkte Rezeption auf wissenschaftlicher, politischer und öffentlicher Ebene verdienen würde. Um dies zu fördern, erscheinen jedoch weniger die hier vorgeschlagenen Änderungen der Indikatoren bzw. des Berichts relevant, sondern eine bessere gesellschaftliche und institutionelle Verankerung wohlstandsorientierter Wirtschaftspolitik allgemein. Eine solche Verankerung würde das Narrativ des WgÖ-Berichts stärken, was wiederum selbstverstärkend auf die Verankerung selbst zurückwirken könnte.

\section{Verknüpfung von Indikatorenset und magischem Vieleck}

Eines der Probleme des magischen Vierecks war neben seiner Beschränkung auf wenige ökonomische Ziele - die fehlende Operationalisierung. Bis heute besteht kein Konsens darüber, wie nun beispielsweise ein außenwirtschaftliches Gleichgewicht genau gemessen wird. Auch was darunter verstanden wurde, änderte sich im Laufe der Zeit (vgl. Dullien/van Treeck 2016). Dieses Problem verschärft sich mit den erweiterten Versionen des magischen Vielecks, die noch mehr qualitative Ziele umfassen.

Eine Verknüpfung aus wirtschaftspolitischen Zielsteuerungssystemen und Wohlstandsindikatoren 
Abbildung 2: Ein operationalisiertes magisches Vieleck wohlstandsorientierter Wirtschaftspolitik

\section{Fair verteilter materieller Wohlstand}

- Arbeitsproduktivität (1)

- Armuts- oder Ausgrenzungsgefährdung (2)

- Hohe/niedrige Bruttojahreseinkommen (3)

- Einkommensverteilung S8o/S20 (3)

- Bruttoinlandsprodukt pro Kopf (4)

- Real verfügbare Haushaltseinkommen (4)

- Hoher/niedriger Haushaltskonsum

- Vermögensverteilung S8o/S2O

\section{Lebensqualität}

- Subjektive Umweltbelastung (2)

- Subjektive Lebenszufriedenheit (2)

- Subjektiver Gesundheitszustand (3)

- Physisches Unsicherheitsempfinden (3)

- Tertiärquote der 30- bis 34-Jährigen (?)

- Vertrauen in das politische System (?)

- Gesellschaftliche Teilhabe

\section{Stabile Finanzmärkte}

- Bilanzsumme des Finanzsektors

- Eigenkapitalquote der Banken

- Private Verschuldung

\section{Preisstabilität}

- Inflationsrate

- Kerninflationsrate

\section{Vollbeschäftigung und gute Arbeit}

- Erwerbstätigenquote (3)

- Arbeitslosenquote (4)

- Freizeit nach Geschlecht (?)

- Nicht bezahlte Arbeit (?)

- Jobzufriedenheit

Quelle: eigene Darstellung.

Anmerkungen: Die Ziffern geben die Bewertung der kurzfristigen Entwicklung des Indikators durch den ExpertInnenbeirat von WgÖ an, wobei die Wettersymbole in ein Schulnotensystem umgewandelt wurden. Nicht bewertete Indikatoren sind mit einem Fragezeichen versehen. Indikatoren in kursiver Schrift sind nicht im WgÖ-Indikatorenset enthalten (und damit zwangsläufig dort nicht bewertet). Einige WgÖ-Indikatoren wurden in den Vorschlag nicht übernommen (Ökosteueranteile, Umweltwirtschaftsumsatz) bzw. durch ähnliche Indikatoren ersetzt (durchschnittlicher Konsum privater Haushalte, Tragfähigkeit sozialer Beziehungen).

kann dieses Problem zu einem Gutteil lösen. Das WgÖ-Indikatorenset erscheint hierfür ein geeigneter Ausgangspunkt. Die beiden großen Bereiche Lebensqualität und umweltorientierte Nachhaltigkeit sind praktisch ident mit zwei Zielen des magischen Vielecks in der AK-Variante. Materieller Wohlstand gemäß WgÖ umfasst eine materielle Dimension sowie das Verteilungsziel des magischen Vielecks. Diese Dimension lässt sich für eine neue VieleckVersion nutzen, indem die beiden Ziele Wirtschafts- wachstum und faire Verteilung zu einem neuen Ziel „fair verteilter materieller Wohlstand“ zusammengefasst werden. Als viertes Ziel kann „Vollbeschäftigung“ um "gute Arbeit“ (vgl. auch IG-Metall 2007) erweitert werden, um expliziter die Arbeitswelt zu adressieren bzw. den zunehmend wichtigen qualitativen Anforderungen gerecht zu werden. Auch hierfür kann direkt auf das WgÖ-Indikatorenset zurückgegriffen werden - für das Vieleck bedarf es lediglich einer Neugruppierung. 
Zur Operationalisierung der instrumentellen wirtschaftspolitischen Ziele des magischen Vielecks, die als indirekte Ziele einer anderen Logik folgen als das WgÖ-Indikatorenset, bedarf es ebenfalls Indikatoren. Das betrifft erstens die Preisstabilität, die innerhalb der Eurozone mit einer Inflationsrate von nahe aber unter $2 \%$ bereits definiert wurde. Zudem sollte die politisch relevante Größe der Kerninflationsrate aufgenommen werden. Zweitens fehlen Indikatoren für eine langfristig stabile Staatstätigkeit. Auf europäischer Ebene ist diese einseitig durch eine Vielzahl restriktiver Fiskalregeln definiert, die sich jedoch nicht bewährt haben, insbesondere weil sie in der Krise zu kontraproduktiven Kürzungen ohne Rücksicht auf die langfristige Leistungsfähigkeit des Staates geführt haben (vgl. Heimberger 2016). In Anlehnung an Dullien und van Treeck (2012) wird deshalb vorgeschlagen, eine Kombination aus langfristiger Verschuldungsregel (strukturelles Defizit) und Nettoinvestitionen als Maßstab für die Entwicklung von Leistungsfähigkeit zu verwenden. Mit einer goldenen Investitionsregel, die in der operationalisierten Variante von Truger (2015) sowohl den strukturellen Saldo als auch die Nettoinvestitionen erfasst, könnte diese ausgewogene Betrachtung sogar relativ leicht als wirtschaftspolitische Orientierungsgröße institutionell verankert werden. Mittelfristig sollten qualitative Indikatoren zur Leistungserbringung des öffentlichen Sektors das Set ergänzen. Drittens fehlt das außenwirtschaftliche Gleichgewicht. Hierbei wird meistens auf den Leistungsbilanzsaldo abgezielt (auf europäischer Ebene gilt ein Zielwert von +6 und $-4 \%$ des BIP, während Dullien und van Treeck $+/-3 \%$ des BIP vorschlagen). Nachdem das Ziel jedoch eigentlich die Bestandsentwicklung betrifft (eine zu stark steigende Auslandsverschuldung und ein spiegelbildlich destabilisierendes bzw. von Abschreibung bedrohtes Wachstum der Auslandsvermögen), wäre auch hier eine strukturelle Zielgröße $\mathrm{zu}$ bevorzugen. Erste Ansätze lassen sich beispielsweise am OFCE in Frankreich finden (wenn auch lediglich in Bezug auf die Handelsbilanz, vgl. Timbeau et al. 2016). Zudem sollten in Hinblick auf die globalen UN-Entwicklungsziele auch die Ausgaben für Entwicklungszusammenarbeit als Indikator aufgenommen werden. Über das magische Vieleck der AK hinausgehend sollte in Anbetracht der Finanzkrise, die zur großen Rezession führte, das instrumentelle Ziel „stabile Finanzmärkte“ aufgenommen werden, operationalisiert beispielsweise durch die Indikatoren Bilanzsumme des Finanzsektors (in $\%$ des BIP), private Verschuldung (in \% des verfügba- ren Einkommens) und Eigenkapitalquote der Banken (Verhältnis Eigenkapital zur Bilanzsumme).

Ein solches wirtschaftspolitisches Steuerungssystem aus Zielen und Indikatoren zu deren Messung wäre eine wichtige Grundlage für eine ausgewogene wohlstandsorientierte Wirtschaftspolitik. Ausgehend vom Niveau sowie der zeitlichen Entwicklung der Indikatoren wäre es leichter, einen Konsens für Prioritäten herzustellen. Interessen- und Zielkonflikte würden dadurch zwar nicht aufgehoben, aber transparenter, sodass Widersprüche auf sachorientierter Basis besser bearbeitbar wären. Wichtige Aufgabe der Politik ist es dann, eine mehrheitsfähige Prioritätensetzung zu entwickeln - ohne die Illusion zu wecken, gleichzeitig alle Ziele zur vollsten Zufriedenheit aller erreichen zu können. Die wirtschaftspolitische Steuerungsarchitektur kann dabei durch eine strukturelle Verankerung der Debatte inklusive demokratischer Entscheidungsfindung unter Einbeziehung möglichst aller relevanten Akteure unterstützend wirken (vgl. Vorschläge im folgenden Abschnitt).

Auch wenn explizite Zielwerte für die Indikatoren des operationalisierten magischen Vielecks wohlstandsorientierter Wirtschaftspolitik ebenso fehlen wie repräsentative Umfragen zur gesellschaftlich aggregierten subjektiven Bedeutung der einzelnen Ziele, ist davon auszugehen, dass sich in einer indikatorengestützten gesellschaftlichen Debatte über eine zielführende wohlstandsorientierte Prioritätensetzung das Ziel „Vollbeschäftigung und gute Arbeit“ als aktuell oberste Priorität durchsetzen dürfte. Auch wenn es einer genaueren Analyse bedürfte, so scheint bei einer oberflächlichen Betrachtung der Indikatoren angesichts der Rekordarbeitslosigkeit die Abweichung vom Ziel Vollbeschäftigung besonders groß. Auch hinsichtlich des Ziels der intakten Umwelt deuten einige Indikatoren (Flächeninanspruchnahme, energetischer Endverbrauch) auf einen besonderen Handlungsbedarf hin, ebenso beim Verteilungs- und Wohlstandsziel (Niveau des Gender-Pay-Gap, Verteilung der Freizeit nach Geschlecht, Einkommens- und Vermögensverteilung).

Bei der Erreichung der Ziele „stabile Staatstätigkeit“, „Preisstabilität“ und „außenwirtschaftliches Gleichgewicht" ist die jüngste Entwicklung sowohl auf österreichischer wie auch auf europäischer Ebene überschießend. Hier bestehen derzeit sogar zusätzliche wirtschaftspolitische Spielräume: Die Inflation erreicht nicht einmal das europäische Ziel von nahe, aber unter $2 \%$, und der Eurozone-Leistungsbilanzüberschuss 
von knapp $4 \%$ des BIP erlaubt viel mehr Importe, als dies derzeit der Fall ist. Die Staatsfinanzen sind in Österreich nicht nur stabil, sondern wiesen in der für die europäischen Fiskalregeln relevanten Frühjahrsprognose der EU-Kommission für 2015 mit einem strukturellen Überschuss sowohl vor (o,4 \% des BIP) als auch nach Nettoinvestitionen (0,03 \% des BIP) eine Übererfüllung der restriktiven Fiskalregeln auf. Für das Ziel einer stabilen Staatstätigkeit besteht allerdings trotzdem Handlungsbedarf. Das gilt zwar nicht für den sich wieder verschlechternden Ausblick beim strukturellen Budgetsaldo, sondern hinsichtlich der Investitionstätigkeit sowie einer nachhaltigen Verbesserung öffentlicher Leistungen.

Diese Analyse gibt erste Anhaltspunkte für wirtschaftspolitische Maßnahmen, die im Sinne einer wohlstandsorientierten Wirtschaftspolitik gesetzt werden sollten (vgl. Abschnitt 5).

\section{Gesellschaftliche Verankerung wohlstandsorientierter Wirtschaftspolitik}

Einer der Gründe, warum wohlstandsorientierte gegenüber wachstumsorientierten Wirtschaftspolitiken zu kurz kommen, ist ihre mangelnde Verankerung. In „Marktgesellschaften“ ist der Wachstumszwang inhärent, sodass es starker demokratischer Korrekturmechanismen bedarf, um ein gesellschaftlich wünschenswertes Ergebnis zu erzielen bzw. den „downfall of a civilization“ zu vermeiden (vgl. Polanyi 1944/2001: 4).

Aktuell ist eine wohlstandsorientierte Wirtschaftspolitik gesellschaftlich kaum verankert, weder auf europäischer Ebene (vgl. Timbeau et al. 2016: 82ff.) noch innerhalb Österreichs (vgl. Griesser/Brand 2016). In Zeitungen und Fernsehen dominieren die insbesondere auf das Wirtschaftswachstum fokussierenden Prognosen der Wirtschaftsforschungsinstitute die ökonomische Berichterstattung, da sie die für die politischen Weichenstellungen relevanten Zukunftserwartungen enthalten. In wirtschaftspolitischen Debatten wird unter anderem deshalb nicht nur von EntscheidungsträgerInnen einseitig auf Wachstum fokussiert. Die gesamtwirtschaftliche Ausrichtung ist hingegen kaum ein Thema und auch nicht institutionell abgesichert. Neben dem wenig bedeutenden Wirtschaftsbericht der Bundesregierung finden sich Hinweise über die gesamtwirtschaftliche Ausrichtung am ehesten in Dokumenten für die europäische Ebene (z. B. nationales Reformprogramm) oder im Rahmen der Budgetpolitik. Letzteres Feld sorgt auch für eine der größten Governance-Reformen in der jüngeren Vergangenheit, der Bundeshaushaltsrechtsreform. Im Zuge dessen wurde die Wirkungsorientierung eingeführt, die die Ausrichtung der Teilhaushalte bzw. neuer politischer Maßnahmen auf bestimmte Ziele stärken sollte, auch wenn die Ausrichtung des Regierungshandelns insgesamt auf gesamtwirtschaftliche Ziele nicht Teil der Reform war.

Über die inhaltlichen Ziele mit operationalisierten Indikatoren hinaus bedarf es deshalb Prozesse, Institutionen und eines alternativen wirtschaftspolitischen Diskurses, um die Wirtschaftspolitik verstärkt auf eine Wohlstandsorientierung auszurichten (ebd.). Als „Referenzfolie“ für die Diskussion über eine erfolgreiche Verankerung einer wohlstandsorientierten Wirtschaftspolitik (VWW) können zum Teil die Reformen der europäischen wirtschaftspolitischen Governance (REWG) dienen, allerdings mit zwei prinzipiellen Einschränkungen.

Das betrifft erstens den zu engen Prozess der Ausgestaltung der REWG, die im Wesentlichen von demokratisch bestenfalls schwach legitimierten Akteuren hinter verschlossenen Türen ausgearbeitet wurden (vgl. Feigl 2014) und für eine VWW kontraproduktiv wären. Auch der laufende Prozess hat mit demokratisch etablierten Verfahren wenig zu tun, sondern lässt sich in Anlehnung an Colin Crouch (2004/2008) besser als „postdemokratisch“ beschreiben. Die tatsächliche politische Partizipationsmöglichkeit wird eingeschränkt, beispielsweise indem die spezifische Themensetzung selbst nicht mehr zur Diskussion steht (Wettbewerbsorientierung, Fiskalregelerfüllung) und politische Entscheidungen nicht mehr breit diskutiert werden, sondern von ExpertInnen auf Basis scheinbar neutraler wissenschaftlicher Evidenz möglichst automatisch getroffen und von der Politik nur mehr exekutiert werden (Strukturreformen, Pensionsautomatismen etc.). Gerade weil eine indikatorenbasierte wohlstandsorientierte Wirtschaftspolitik Gefahr läuft, diese postdemokratische Tendenz zu reproduzieren, muss ein möglichst breiter Diskussionsprozess bewusst in den Mittelpunkt gerückt werden.

Zweitens sind ähnlich starre Regeln und Verfahren bei Zielverfehlungen zu vermeiden, da diese sowohl aus demokratischen als auch aus keynesianisch geprägten wirtschaftspolitischen Überlegungen kontraproduktiv wären. Sinnvoller als starre Regeln sind mittelfristige Orientierungsgrößen und politische Richtungsbestimmung, einerseits um eine möglichst breite demokratische Debatte anzuregen, anderer- 
Abbildung 3: Vorschläge für eine Verankerung wohlstandsorientierter Wirtschaftspolitik (VWW) vor der Referenzfolie der Reformen der europäischen wirtschaftspolitischen Governance (REWG)

\begin{tabular}{|c|c|c|}
\hline Aspekt & REWG & $V W W$ \\
\hline kohärenter wirtschaftspolitischer Prozess & $\begin{array}{l}\text { Europäische Semester, u. a. mit Prioritäten für das } \\
\text { kommende Jahr, länderspezifische Empfehlungen } \\
\text { und „Genehmigung" von Budgetplänen }\end{array}$ & $\begin{array}{l}\text { Prioritäten nach breitem parlamentarischem } \\
\text { Entscheidungsprozess unter Einbindung von } \\
\text { Sozialpartnern und Zivilgesellschaft }\end{array}$ \\
\hline Indikatorenset & $\begin{array}{l}\text { Scoreboard zur Vermeidung von makroökonomis- } \\
\text { chen Ungleichgewichten }\end{array}$ & Operationalisiertes magisches Vieleck \\
\hline $\begin{array}{l}\text { Richtwerte für besonders wichtige Indi- } \\
\text { katoren und vordefinierte Prozesse zum } \\
\text { Umgang mit Überschreitungen }\end{array}$ & $\begin{array}{l}\text { Insbesondere Regeln des Stabilitäts- und Wachs- } \\
\text { tumspaktes und Androhung von Sanktionen }\end{array}$ & $\begin{array}{l}\text { Fokus auf flexiblere Orientierungsgrößen mit der } \\
\text { Konsequenz eines breiten Diskussionsprozesses }\end{array}$ \\
\hline beratende Institutionen & $\begin{array}{l}\text { Fiskalräte und Produktivitätsausschüsse als } \\
\text { parademokratische ExpertInnengremien }\end{array}$ & $\begin{array}{l}\text { An Parlament und Öffentlichkeit rückgebundene } \\
\text { repräsentative Wohlstands- und Konvergenzräte }\end{array}$ \\
\hline Analyseinstrumente & Jahreswachstumsbericht, Länderberichte & $\begin{array}{l}\text { Jahreswohlstandsberichte auf europäischer, } \\
\text { nationaler und regionaler Ebene }\end{array}$ \\
\hline
\end{tabular}

Quelle: eigene Darstellung.

seits um auch die Erwartungen der wirtschaftlichen Akteure zu stabilisieren - und damit ihre Handlungen, die selbst für den wirtschaftlichen Verlauf relevant sind (beispielsweise die Investitionstätigkeit der Unternehmen). Insbesondere die instrumentellen Ziele wie stabile Staatstätigkeit oder Preisstabilität müssen gemeinsam betrachtet und situationsadäquat bewertet werden, andernfalls führen sie zu extrem komplexen (weil alle Eventualitäten berücksichtigenden) Regeln oder zu besonders negativen Konsequenzen in bestimmten Situationen. So war beispielsweise die regelgebundene europäische Wirtschaftspolitik in den vergangenen Jahren unpassend (vgl. Kitzmantel 2016), weil die starre Fokussierung auf Preis- und Staatshaushaltsziele zu einer für die Entwicklung von Beschäftigung und materiellem Wohlstand kontraproduktiven Politik geführt hat.

Auch wenn die REWG also nicht unproblematisch ist, können mehrere Aspekte trotzdem als richtungsweisend für eine wirkungsvolle VWW gesehen werden:

Ähnlich dem makroökonomischen „Scoreboard“ könnte das operationalisierte magische Vieleck wohlstandsorientierter Wirtschaftspolitik (vgl. Abschnitt 3) ergänzt um qualitative Analysen auf europäischer Ebene als Steuerungssystem mit Kennzahlen wirtschaftspolitische Entscheidungen unterstützen (vgl. Timbeau et al. 2016). Vermieden werden sollte jedoch die Art der Erstellung, die im Europäischen Semester durch scheinobjektive KommissionsbeamtInnen ohne Einbeziehung von Stakeholdern bzw. mit mangelnder demokratischer Legitimierung erfolgt.
Als neue Institution könnte ein „Wohlstandsrat“ analog zu den Fiskalräten oder zum deutschen Sachverständigenrat geschaffen werden. Seine Aufgabe wäre es, anknüpfend am operationalisierten magischen Vieleck, wissenschaftliche Expertise zu liefern und diese in den wirtschaftspolitischen Prozess einzuspielen, beispielsweise indem die Regierung Stellung beziehen muss und das Parlament sich damit befasst. Ein Ansatzpunkt zu seiner Errichtung wären die kürzlich beschlossenen sogenannten Produktivitätsausschüsse (vgl. ECOFIN 2016). Nachdem deren Mandat momentan aber ähnlich einseitig ist wie jenes der Fiskalräte, sollte es erweitert werden, sodass auf eine breite Wohlstandsorientierung und europäische Konvergenz (vgl. Timbeau et al. 2016) fokussiert werden kann. Bei der Bestellung der Mitglieder müsste darauf geachtet werden, dass dieser Wohlstandsrat ausreichend groß und repräsentativ ist, um einen gesellschaftlichen Interessenausgleich $\mathrm{zu}$ ermöglichen (eventuell in Anlehnung an den bereits etablierten Stakeholder-Prozess der Statistik Austria zu WgÖ).

Punkto Prozeduren bedarf es eines Steuerungsprozesses als Pendant zum Europäischen Semester. Nachdem der Bericht der Statistik Austria jeweils im Herbst ungefähr zeitgleich mit dem Start des Europäischen Semesters selbst vorliegt, könnte hier eine enge Verzahnung erfolgen (beispielsweise Bericht des Wohlstandsrates parallel zum Länderbericht der Kommission und Stellungnahme der Regierung als Basis für mittelfristige Budgetplanung und das nationale Reformprogramm). 
Neben der politischen Ebene bzw. über die europäische Economic Governance hinausgehend ist eine verstärkte Wohlstandsorientierung auch im medialen Diskurs und in der (Wirtschafts-)Wissenschaft erforderlich (vgl. Griesser/Brand 2016). Eine Reform der Wirtschaftsprognosen durch die Wirtschaftsforschungsinstitute könnte hierbei auf beiden Ebenen Wirkung entfalten. Relativ leicht umzusetzen wäre die Prognose jener Wohlstandsindikatoren, die auf den Volkswirtschaftlichen Gesamtrechnungen basieren (z. B. verfügbare Einkommen pro Kopf), bei anderen Indikatoren sollte interdisziplinär kooperiert werden. Im Rahmen des WgÖ-Projekts der Statistik Austria könnte zudem der ExpertInnenbeirat aufgewertet werden, der bereits jetzt die kurz- und langfristige grobe Entwicklungsrichtung einschätzt.

Auf Mikroebene könnten zudem die ersten Ansätze alternativer Rechnungslegungssysteme sowohl für Unternehmen als auch für öffentliche oder gemeinnützige Organisationen (vgl. CSR- bzw. GemeinwohlÖkonomie-Ansätze, vgl. Felber 2010) zum Zweck einer breiteren Wohlstands- anstelle der einseitigen Gewinnorientierung unterstützend wirken, auch wenn sie gesamtwirtschaftlich nicht verallgemeinerbar erscheinen.

Als ein bedeutsames Hindernis für eine verstärkte gesellschaftliche Verankerung einer verstärkten Wohlstandsorientierung in der Wirtschaftspolitik erscheinen jedoch die politischen Kräfteverhältnisse. Einen ersten Schritt könnten mehrheitsfähige wohlstandsorientierte Einstiegsprojekte darstellen, wie sie nachfolgend skizziert werden.

\section{Einstiegsprojekte für eine wohlstandsorientierte Wirtschaftspolitik}

Neben diesen Reformen zur Steuerung der Wirtschaftspolitik allgemein gilt es politische Maßnahmen zu setzen, die langfristig eine nachhaltige sozial-ökologische Transformation möglichst effektiv unterstützen und kurzfristig vor allem an jenen Zielen des magischen Vielecks ansetzen, die derzeit besonderen Handlungsbedarf aufweisen. Welche Ziele das genau sind, muss zwar im Rahmen von gesellschaftlichen Aushandlungsprozessen festgelegt werden, doch liefern die wirtschaftspolitischen Indikatoren (vgl. Abschnitt 3) recht klare Anhaltspunkte für eine zukünftige Prioritätensetzung.

Gesucht sind wirtschaftspolitische Maßnahmen, die insbesondere die Arbeitslosigkeit reduzieren, die ökologische Krise entschärfen und eine fairere Verteilungssituation schaffen - ohne die staatlichen Haushalte übermäßig zu belasten. Ein Beispiel für ein interessantes Bündel an konkreten sozial- und wirtschaftspolitischen Vorschlägen mit diesen Prioritäten findet sich in Österreich im jährlichen „Zukunftsbudget" der Initiative „Wege aus der Krise“, einer Allianz aus etwa einem Dutzend zivilgesellschaftlicher Organisationen und noch mehr unterstützenden Vereinen (vgl. Wege aus der Krise 2016).

In Folge werde ich auf vier prioritäre Maßnahmenbündel fokussieren, die mir sowohl situativ wie auch langfristig hinsichtlich einer ausgewogeneren, wohlstandsorientierten Wirtschaftspolitik im Sinne der Ziele des magischen Vielecks besonders vielversprechend erscheinen. Sowohl ein Ausbau sozialer Dienstleistungen (5.1) als auch eine verstärkte öffentliche Investitionstätigkeit (5.2) können die Arbeitslosigkeit reduzieren und - da in Österreich vor allem über die Ausgabenseite des Staates umverteilt wird (vgl. Rocha-Akis et al. 2016) - die Verteilungssituation verbessern. Abhängig von der genauen Verwendung der Investitionen kann zudem die ökologische Nachhaltigkeit entscheidend verbessert werden. Arbeitszeitverkürzung (5.3) und die globale Absicherung höherer Sozial- und Umweltstandards (5.4) würde vor allem die Erreichung der Ziele „Vollbeschäftigung und gute Arbeit“, „Lebensqualität“ und „umweltbezogene Nachhaltigkeit“ unterstützen.

\subsection{Ausbau sozialer Dienstleistungen finanziert} durch umwelt- und vermögensbezogene Steuern

Als prioritäre Maßnahme geeignet erscheint der weitere Ausbau sozialer Dienstleistungen, gegenfinanziert durch höhere vermögens- und umweltbezogene Steuern. Darunter fällt die Ausweitung der Kinderbetreuung, Ganztagesschulen, Bildungsangebote, Sozialarbeit und Pflege. Soziale Dienstleistungen sind sehr beschäftigungsintensiv, sodass zusätzliche Ausgaben von 1 Mrd. Euro mit einem Beschäftigungseffekt von 20.000 (Marterbauer 2011: 141) bis langfristig 45.000 (vgl. Kurzmann/Gstinig 2012: 107) Personen einen großen Beitrag zur Reduktion der Arbeitslosigkeit leisten können, insbesondere jener von Frauen. Sie ermöglichen gleichzeitig eine Reduktion der unbezahlten Arbeit bzw. ihre gleichere Verteilung zwischen den Geschlechtern.

Langfristig tragen sie dazu bei, die sozialen wie öffentlichen Kosten zu senken. Beispielsweise ist es besser, durch verstärkte Bildungsangebote bzw. durch eine Intensivierung der Integrationsangebote oder mehr Sozialarbeit heute den sozialen Problemen von morgen vorzubeugen. 
Auswirkungen auf die Umwelt gibt es lediglich indirekt durch den Einkommenseffekt. Werden gleichzeitig umwelt- und vermögensbezogene Steuern erhöht, ist der Nettoeffekt sehr wahrscheinlich positiv, auch weil besonders ressourcenintensiver Konsum der reichen Haushalte (OXFAM 2015) gedämpft werden könnte.

5.2 Öffentliche Investitionen (v. a. in Wohnbau, Forschung und Mobilität)

Die Ökologisierung und Digitalisierung unserer Lebensweise und der anhaltende Zuzug in den Ballungsräumen erfordern mehr Investitionen als das derzeit der Fall ist, insbesondere in den Bereichen sozial-ökologischer Wohnbau, Energienetze, Forschung und öffentlicher Verkehr. Mehr öffentliche Investitionen schaffen auch Beschäftigung. Wie private Investitionen sollen auch öffentliche Investitionen kreditfinanziert getätigt werden können, wofür eine Änderung der selbstlähmenden Fiskalregeln auf nationaler wie europäischer Ebene notwendig ist. Im Sinne der Generationengerechtigkeit sind diese höheren Ausgaben gerechtfertigt, da ihnen auch höhere zukünftige Werte und Erträge gegenüberstehen, von denen zukünftige Generationen profitieren. Das gilt umso mehr in Zeiten extrem niedriger Zinsen, die die Finanzierungskosten dieser Zusatzausgaben drücken - und damit ihre langfristige Rentabilität steigern.

Der Transport von Menschen und Gütern, der nach wie vor stark auf fossilen Energiequellen basiert, ist als ein wesentlicher belastender Faktor für das Weltklima rasch nachhaltiger zu gestalten. Tendenziell muss die derzeitige Form des Individual- und Güterverkehrs reduziert und öffentlicher Verkehr bzw. auf nichtmotorisierten oder durch erneuerbare Energien angetriebenen Individualverkehr ausgeweitet werden. Ähnliches gilt für eine höhere Energieeffizienz, insbesondere bei Gebäuden.

\subsection{Arbeitszeitverkürzung}

Obwohl das Arbeitsvolumen in Österreich kaum mehr wächst, wird ständig mehr produziert und auch konsumiert - bei steigender Arbeitslosigkeit. Arbeitszeitverkürzung kann nicht nur die bezahlte Arbeit gerechter verteilen, sondern ermöglicht jenen, die Arbeit haben, steigenden Wohlstand auch in Zeitwohlstand umzusetzen. Außerdem ermöglicht sie es Familien, die Zeit der Eltern mit den Kindern besser zwischen den Geschlechtern zu verteilen.
Eine Vorbedingung gelungener Arbeitszeitverkürzung sind innovative Formen der Arbeitsorganisation - vor allem dann, wenn von der 40-Stunden-fünf-Tage-Woche abgerückt wird (vgl. Poyntner 2016). Eine besondere soziale Innovation der letzten Jahre stellt die sogenannte Freizeitoption in manchen Kollektivverträgen dar (vgl. Marterbauer 2014), bei der die Beteiligung an Produktivitätszuwächsen nicht mehr nur in Form von Lohnzuwächsen erfolgt, sondern die ArbeitserbringerInnen sich auch für mehr Freizeit entscheiden können.

Eine Verkürzung überlanger Arbeitszeiten durch stärkere Kontrolle der Arbeitsgesetze, Anreize zum Abbau von Überstunden (Erhöhung der Überstundenzuschläge, Streichung der Steuerermäßigung für Überstunden) und Einschränkung von Umgehungskonstruktionen wie All-In-Verträgen sind weitere Möglichkeiten. Positiver Zusatzeffekt ist eine Reduktion der stressbedingten Erkrankungen und höhere Lebensqualität durch bessere „Work-Life-Balance“. Auch eine Ausweitung des Urlaubsanspruchs, die Erleichterung von Elternteilzeit, Sabbaticals, Qualifizierungsstipendien und Bildungskarenzen eröffnet neue Möglichkeiten für Beschäftigung und Lebensqualität.

5.4 Globale Expansion von Sozial- und Umweltstandards statt bedingungslosem Wettbewerb

Der Paradeeinwand gegen ein besseres Leben für alle ist, dass „wir“ uns das nicht leisten können, wenn wir im internationalen Wettbewerb bestehen wollen. In der Tat gilt, dass je kleiner und vernetzter das Gebiet ist, in dem besondere soziale Rechte, ökologische Standards, progressive Abgaben, soziale Dienstleistungen und hochwertige öffentliche Infrastruktur umgesetzt werden, desto stärker ist der Druck, dass diese Regulierung auf das niedrigere Niveau anderer Regionen reduziert wird. Relativierend ist aber festzuhalten, dass in vielen Fällen höhere soziale und ökologische Standards kein Widerspruch zur internationalen Wettbewerbsfähigkeit sind, da sie technologische Spitzenleistungen eher befördern, die für die Wettbewerbsfähigkeit relevanter sind als die Kostenentwicklung.

Das vierte prioritäre Handlungsfeld ist deshalb die bessere Verankerung von sozial-ökologischen Standards durch internationale bzw. idealerweise globale Kooperation - auch wenn dort die Kräfteverhältnisse für eine sozial-ökologische Transformation noch schlechter sind als auf nationaler Ebene. Ähnliches gilt auch für die effektive Einhebung von Steuern nach dem Leistungsfähigkeitsprinzip. 
Mit der Europäischen Union wurde die Möglichkeit eines riesigen Regulationsraumes geschaffen, der wirtschaftlich betrachtet nach wie vor sehr geschlossen ist. Knapp 90 \% der in der EU erzeugten Waren und Dienstleistungen (vgl. Feigl 2014: 34) werden innerhalb der Europäischen Union selbst abgesetzt. Deshalb muss es gelingen, zunächst hier hohe Standards durchzusetzen, da die transnationale Kooperation relativ leicht möglich ist.

Darüber hinaus ist eine andere Handelspolitik gefragt (vgl. Kapeller et al. 2015). Diese darf nicht länger die Konkurrenz um den Abbau von Standards in den Exportmärkten anheizen, sondern muss umgekehrt ein Instrument der transnationalen Kooperation werden. So ließen sich hohe eigene sozial- und ökologische Standards nicht nur absichern, sondern auf globaler Ebene expandieren - ganz im Sinne eines besseren Lebens für wirklich alle.

\section{Zusammenfassung}

Gesellschaftlicher Fortschritt bedarf einer Wirtschaftspolitik, die - unter Berücksichtigung ökologischer Grenzen - auf Wohlstand als wichtigem Beitrag für ein gutes Leben für alle fokussiert. Angesichts einer zunehmenden Verengung des öffentlichen Diskurses auf Wachstum und Wettbewerbsfähigkeit - in Österreich wie in Europa - ist dieses Vorhaben voraussetzungsvoll. So wie im Zuge der Reformen der European Economic Governance (Feigl 2014) in der EU Wettbewerbsfähigkeit in Kombination mit einer auf Staatsabbau gerichteten Austeritätspolitik als Leitziele verfestigt wurden, bedarf wohlstandsorientierte Wirtschaftspolitik einer institutionellen Verankerung.

Das Konzept eines neuen magischen Vielecks der Wirtschaftspolitik kann diesen Prozess unterstützen, da Zielkonflikte transparenter werden und so eher gesellschaftlich ausgehandelt werden können. In Kombination mit einer objektivierten Messung von Wohlstand bzw. der Ziele des Vielecks können die Grundlagen für eine auf gesellschaftlichen Interessenausgleich basierende Wirtschaftspolitik verbessert werden, für die Österreich bekannt ist (vgl. Tálos 2015). Nicht zuletzt die Mehrheit in der Volksabstimmung für einen EU-Austritt in Großbritannien, die vor allem auf die fehlende Berücksichtigung des schlechter gestellten Teils der Bevölkerung zurückzuführen war, zeigt, dass die Förderung eines verstärkten Interessenausgleichs auch für die europäische Ebene gefordert wäre.
Mit der Initiative „Wie geht's Österreich?“ der Statistik Austria ist hierzulande bereits eine gute Ausgangslage geschaffen - die jedoch ohne Einbettung in die Wirtschaftspolitik und die gesellschaftliche Debatte unzureichend für gesellschaftlichen Fortschritt bleiben muss (vgl. Griesser/Brand 2016). Eine erfolgreiche Verankerung von Zielen und Erfolgsindikatoren wohlstandsorientierter Wirtschaftspolitik wird die Durchsetzungsfähigkeit wirtschaftspolitischer Maßnahmen, die besonders effektiv zu Wohlstand und sozialem Fortschritt beitragen, strukturell verbessern. Ausgehend von einer indikatorenbasierten Bewertung der Zielerreichung wohlstandsorientierter Wirtschaftspolitik sind aktuell vor allem jene Maßnahmen voranzutreiben, die die Beschäftigung steigern, die ökologische Krise entschärfen und eine fairere Verteilungssituation schaffen - ohne die staatlichen Haushalte massiv zu belasten. Diese Kriterien treffen insbesondere auf einen Ausbau sozialer Dienstleistungen finanziert durch umwelt- und vermögensbezogene Steuern, mehr öffentliche Investitionen (v. a. in Wohnbau, Forschung und ökologischere Mobilität), Arbeitszeitverkürzung und der globalen Expansion von Sozial- und Umweltstandards zu.

Ansätze einer Neuorientierung auf Wohlstand und sozialen Fortschritt unter Berücksichtigung ökologischer Grenzen gilt es nun gesellschaftlich sowohl auf europäischer als auch nationaler Ebene zu verankern - insbesondere mit neuen Zielsystemen, neuen Indikatoren und neuen wirtschaftspolitischen Maßnahmen.

\section{Literatur}

Aiginger, K. (2016): New Dynamics for Europe: Reaping the Benefits of Socio-Ecological Transition. Online: http:// www.foreurope.eu/fileadmin/documents/pdf/Deliverables/WWWforEurope_Synthesis_Report_Part_I_Executive_Summary_D602.5.pdf [8.8.2016].

BEIGEWUM (2015): Politische Ökonomie Österreichs. Wien: Mandelbaum.

Chaloupek, G./Feigl, G. (2012): Die Wachstumskontroverse vor 40 Jahren und heute. Wirtschaft und Gesellschaft 4/2012, 771-800.

Chaloupek, G./Marterbauer, M. (2008): Was bleibt vom Austrokeynesianismus? Dauerhafte Wirkungen der postkeynesianischen Wirtschaftspolitik in Österreich 1970-1995. In: Hagemann, H./Horn, G./Krupp, H. J. (Hg.): Aus gesamtwirtschaftlicher Sicht. Festschrift für Jürgen Kromphardt. Marburg: Metropolis, 45-67.

Crouch, C. (2004/2008): Postdemokratie. Frankfurt am Main: Suhrkamp. 
Deutscher Bundestag (2013): Enquete-Kommission „Wachstum, Wohlstand, Lebensqualität - Wege zu nachhaltigem Wirtschaften und gesellschaftlichem Fortschritt in der Sozialen Marktwirtschaft". Online: http://webarchiv. bundestag.de/archive/2013/1212/bundestag/gremien/enquete/wachstum/index.html [archiviert am 12.12.2013; Zugriff 15.9.2016].

Dullien, S./van Treeck, T. (2016): Reform des Stabilitäts- und Wachstumsgesetzes von 1967. Online: http://library.fes. de/pdf-files/wiso/12501.pdf [8.8.2016].

Dullien, S./van Treeck, T. (2012): Ziele und Zielkonflikte der Wirtschaftspolitik und Ansätze für Indikatoren und Politikberatung. Düsseldorf: IMK.

ECOFIN - Rat (Wirtschaft und Finanzen) der Europäischen Union (2016): Empfehlung für eine EMPFEHLUNG DES RATES zur Einrichtung nationaler Ausschüsse für Produktivität. Online: http://data.consilium.europa.eu/doc/ document/ST-10083-2016-INIT/de/pdf [8.8.2016].

Europäische Kommission (2001): Europäisches Regieren - ein Weissbuch. Online: http://eur-lex.europa.eu/LexUriServ/LexUriServ.do?uri=CELEX:52001DC0428:DE:PDF [8.8.2015].

Europäische Kommission (1993): Wachstum, Wettbewerbsfähigkeit, Beschäftigung. Herausforderungen der Gegenwart und Wege ins 21. Jahrhundert. Weißbuch. Luxemburg: Amt für amtliche Veröffentlichungen der Europäischen Gemeinschaften.

Eurostat (2016): Übersicht „BIP und mehr“. Online: http:// ec.europa.eu/eurostat/de/web/gdp-and-beyond [15.9.2016]

Feigl, G. (2015): Nationale Wettbewerbsräte als problematischer Ausdruck europäischer Wettbewerbsfixierung. Online: http://blog.arbeit-wirtschaft.at/eu-wettbewerbsfixierung-reloaded [1.8.2016].

Feigl, G. (2014): The New Economic Governance: a critical overview. In: Rocha, F. (Hg.): The New EU Economic Governance and ist Impact on the National Collective Bargaining Systems. Madrid: Fundación $1^{\circ}$ de Mayo, 24-38.

Feigl, G./Marterbauer, M./Rossmann, B./Schlager, Ch./ Schweitzer, T. (2014): Budgetanalyse 2014-2018. Wien: AK Wien.

Felber, Ch. (2010): Die Gemeinwohl-Ökonomie. Das Wirtschaftsmodell der Zukunft. Wien: Deuticke.

Griesser, M./Brand, U. (2016): Verankerung Wohlstandsorientierter Politik. Working Paper-Reihe der AK Wien - Materialien zu Wirtschaft und Gesellschaft, Nr. 165 Online: https://emedien.arbeiterkammer.at/viewer/file?pi= AC13397506\&file=AC13397506.pdf [12.1.2017].

Heimberger, P. (2016): Did Fiscal Consolidation Cause the Double-Dip Recession in the Euro Area? wiiw Working Paper N. 130. Online: http://wiiw.ac.at/did-fiscal-consolidation-cause-the-double-dip-recession-in-the-euroarea--dlp-3988.pdf [30.1.2017].

IG-Metall Projekt Gute Arbeit (Hg., 2007): Handbuch „Gute Arbeit". Hamburg: VSA.
Jessop, B. (2014): Kapitalismus, Steuerung und der Staat. Online: http://bobjessop.org/2014/o1/11/kapitalismus-steuerung-und-der-staat. [6.8.2014].

Kapeller, J./Schütz, B./Tamesberger, D. (2015): Von freien zu zivilisierten Märkten. Ein New Deal für die europäische Handelspolitik. Wien: Österreichische Gesellschaft für Europapolitik.

Kitzmantel, E. (2016): EU-Fiskalregeln - Anker oder Mühlstein der europäischen Wirtschaftspolitik? Wirtschaft und Gesellschaft 3/2016, 431-450.

Kurzmann, R./Gstinig, K. (2012): Beschäftigungsmultiplikatoren und die Besetzung von Arbeitsplätzen in Österreich. Online: https://www.joanneum.at/uploads/tx_publicationlibrary/BESCHAEFTIGUNGSMULTIPLKATOREN_IN_OESTERREICH.pdf [8.5.2017].

Marterbauer, M. (2014): Bemerkenswerte Innovation: Die Freizeitoption im Kollektivvertrag. Online: http://blog. arbeit-wirtschaft.at/bemerkenswerte-innovation-diefreizeitoption-im-kollektivvertrag [8.4.2016].

Marterbauer, M. (2011): Zahlen bitte! Die Kosten der Krise tragen wir alle. Wien: Deuticke.

Meichenitsch, K. (2015): Was machen wir mit der Sozialen Innovation? Online: http://www.armutskonferenz.at/files/ meichenitsch_soziale-innovation_10armkon-2015.pdf [1.8.2016].

OECD (2016): Better Life Initiative: Measuring Well-Being and Progress. Online: http://www.oecd.org/statistics/ better-life-initiative.htm [15.09.2016].

OXFAM (2015): Extreme Carbon Inequality. Online: https:// www.oxfam.org/sites/www.oxfam.org/files/file_attachments/mb-extreme-carbon-inequality-021215-en.pdf [15.09.2016].

Polanyi, K. (1944/2001): The Great Transformation. The Political and Economic Origins of Our Time. Boston: Beacon Press

Poyntner, P. (2016): Arbeitszeitverkürzung als Beschäftigungsmotor? Online: http://blog.arbeit-wirtschaft. at/arbeitszeitverkuerzung-als-beschaeftigungsmotor [1.8.2016].

Rocha-Akis, S./Bierbaumer-Polly, J./Einsiedl, M./Guger, A./ Klien, M./Leoni, Th./Lutz, H./Mayrhuber, Ch. (2016): Umverteilung durch die öffentlichen Haushalte in Österreich. Wien: WIFO.

Rothschild, K. W. (1996): Alternative Konzepte der Wirtschaftspolitik, in: Forum Politische Bildung (Hg.): Politik und Ökonomie. Wien: ÖBV, 5-12.

Stiglitz, J./Sen, A./Fitoussi, J.-P. (2009): Report by the Commission on the Measurement of Economic Performance and Social Progress. Online: http://library.bsl.org.au/ jspui/bitstream/1/1267/1/Measurement_of_economic_ performance_and_social_progress.pdf [8.5.2017].

Statistik Austria (2015): Wie geht's Österreich? Indikatoren und Analysen. Wien: Statistik Austria.

Tálos, E. (2015): Austrokorporatismus zwischen Kontinuität und Veränderung - EU-Beitritt als Bestimmungsfaktor 
für Veränderungen. In: BEIGEWUM (Hg., 2015): Politische Ökonomie Österreichs. Wien: Mandelbaum, 176-197.

Timbeau, X./Dahl, S./Feigl, G./Watt, A. (Hg., 2016): The Elusive Recovery. Independent Annual Growth Survey (iAGS) 2017. Working Paper-Reihe der AK Wien - Materialien zu Wirtschaft und Gesellschaft, Nr. 164. Online: https://emedien.arbeiterkammer.at/viewer/image/ AC13385395/1/LOG_0003 [20.12.2016].

Truger, A. (2015): Implementing the Golden Rule for Public Investment in Europe - Safeguarding Public Investment and Supporting the Recovery. Wien: AK Wien.

Wege aus der Krise (2016): Das Zivilgesellschaftliche Zukunftsbudget 2017-2019. Online: http://www.wege-aus-der-krise.at/fileadmin/dateien/Baustelle_2016/Zukunftsbudget_2017_-_2019_web.pdf [30.01.2017]. 